\title{
Comparative analysis of CEACAM1 expression in thin melanomas with and without regression
}

\author{
LUCIANA NICHITA $^{1,2}$, SABINA ZURAC $^{1,2}$, ALEXANDRA BASTIAN $^{1,2}$, PATRICIA STINGA $^{2}$, \\ ROXANA NEDELCU ${ }^{3}$, ALICE BRINZEA ${ }^{3}$, GABRIELA TURCU ${ }^{3,4}$, DANIELA ION ${ }^{3}$, \\ LUCIA JILAVEANU ${ }^{5}$, LIANA STICLARU ${ }^{2}$, CRISTIANA POPP ${ }^{2}$ and MIRELA CIOPLEA ${ }^{2}$
}

\author{
${ }^{1}$ Department of Pathology, Faculty of Dentistry, Carol Davila University of Medicine and Pharmacy, 010221 Bucharest; \\ ${ }^{2}$ Department of Pathology, Colentina University Hospital, 020125 Bucharest; ${ }^{3}$ Department of Physiopathology, \\ Faculty of Medicine, Carol Davila University of Medicine and Pharmacy, 020021 Bucharest; ${ }^{4}$ Department of \\ Dermatology, Colentina University Hospital, 020125 Bucharest, Romania; ${ }^{5}$ Department of Medicine, Section of \\ Medical Oncology, Yale University School of Medicine, New Haven, CT 208028, USA
}

Received September 11, 2018; Accepted December 18, 2018

DOI: 10.3892/ol.2019.10067

\begin{abstract}
Carcinoembryonic antigen-related cell adhesion molecule 1 (CEACAM1) is a key molecule in several intracellular and intercellular signaling pathways, with multiple functional and structural roles. CEACAM1 expression in melanoma is often described in the invading part of the tumor and has been associated with increased melanoma cells invasion and migration. We studied CEACAM1 expression in regressing versus non-regressing thin melanomas, knowing that phenomenon of regression represents a valuable model for understanding tumor immunity. In melanoma, through homophilic interactions, CEACAM1 inhibits natural killer cell activity, inhibits effector functions of tumor infiltrating lymphocytes, such as cytotoxicity and interferon- $\gamma$ release. We present a retrospective study including 53 consecutive cases of thin melanoma, 21 with regression and 32 without regression. Comparative analysis of CEACAM1 expression in regressed and non-regressed areas from melanomas with regression and in non-regressed melanomas was performed. We used three different clones of CEACAM1: AA 1-428, extracellular domain, rabbit; AA 1-428, mouse, clone 8B6E2F4; and AA 1-468, full length, mouse, clone 2F6. All three clones had similar reactivity. We identified membrane positivity of tumor cells in non-regressed melanomas and in non-regressed areas in melanomas with regression. Remaining tumor cells in regressed areas were mostly negative for CEACAM1. In nonregressed lesions, there was a stronger positivity of CEACAM1
\end{abstract}

Correspondence to: Dr Cristiana Popp, Department of Pathology, Colentina University Hospital, 19-21 Stefan cel Mare Street, 020125 Bucharest, Romania

E-mail: brigaela@yahoo.com

Key words: CEACAM1, melanoma, regression, invasion front in the deep invasive front. In thin melanomas, CEACAM1 overexpression is related with invasiveness, suggesting that CEACAM1-positive melanomas are more aggressive. Also, in areas of regression tumor cells lose CEACAM1 expression, probably correlated with the presence of natural killer cells.

\section{Introduction}

Carcinoembryonic antigen-related cell adhesion molecule 1 (CEACAM1), also known as CD66a is a key molecule in several intracellular and intercellular signaling pathways, with multiple functional and structural roles. It is expressed in some normal cells (epithelial cells of large bowel, prostate, bile duct, salivary ducts, endothelial cells, T lymphocytes) and also in several tumors (melanoma, non-small cell lung carcinomas, breast, large bowel, gastric, thyroid carcinoma) (1). CEACAM1 is a transmembrane protein that includes an extracellular $\mathrm{N}$-terminal variable domain and three constant C2-like immunoglobulin domains (2). Its extracellular domain is responsible for homophilic (CEACAM1-CEACAM1) and heterophilic intercellular adhesion with CEA and $\mathrm{T}$ cell immunoglobulin and mucin domain (3).

CEACAM1 expression in melanoma was studied as diagnosis marker, as well as a prognosis and treatment efficacy indicator (4,5). CEACAM1 expression has often been described in the invading part of the tumor and associated with increased melanoma cell invasion and migration and a poor prognosis (4,6-8). Although initially it was identified as a tumor suppressor, with roles in apoptosis and tumorigenesis (9), CEACAM1 demonstrates involvement in a mechanism used by tumor cells to evade the immune system, especially cytotoxic lymphocyte attack (4). In melanoma, through homophilic interactions, CEACAM1 inhibits natural killer (NK) cell activity, as well as immune functions of tumor infiltrating lymphocytes (TILs), such as cytotoxicity and IFN- $\gamma$ release $(3,4,6,10,11)$. Some patients with melanoma have increased serum levels of CEACAM1, described as a factor of poor prognosis, predicting response failure to immunotherapy and tendency to 
early progression and metastasis $(6,12,13)$. These serum levels can also be used in patient surveillance as indicators for tumor progression and treatment response $(6,12,13)$.

For the above reasons, CEACAM1 was identified as potential therapeutic target in melanoma, and some monoclonal antibodies against CEACAM1 were designed and studied in melanoma immunotherapy (14).

Some studies compared CEACAM1 expression in various melanocytic lesions and identified an increased expression correlated with the severity and evolutive potential of the lesion: CEACAM1 was negative in non-tumoral melanocytes, positive in very few common nevi and increasingly more frequenty positive in dysplastic nevi, thin melanomas, thick melanomas and melanoma metastasis $(10,15,16)$.

There are still only a few studies concerning special characteristics of CEACAM1 expression in melanoma and their correlations with other morphologic and immunohistochemical traits of tumors. Especially, in thin melanomas the data can help in stratifying patients according their risk to progression and poor outcome, since there is a significant variability of these patients evolution (17).

On the other hand, regression in melanoma is a studied feature, with controversial biologic potential. It is defined as decrease of the number of tumor cells accompanied by stromal reaction (fibrosis, inflammatory infiltrate with variable number of melanophages, capillaries ectasias), determined by the immune response of the host $(18,19)$. Some studies credited regression with an aggressive comportment, and, although it is not included in staging, there are opinions supporting the idea that presence of histological confirmed regression is an indicator for sentinel node biopsy in patients with thin melanomas $(17,18)$. Multiple efforts were made to identify the best way to characterize regression in order to make it a reproducible routine feature to be reported by pathologists (20). Three types of regression (complete, segmentary and partial) have been described with different incidence and different impact on patient outcome (20). Globally, regression is present in about half of melanomas, being less frequent in thick melanomas (20).

Similar lesions occur in benign melanocytic tumors ('halo nevi') but there are evident morphologic differences with areas of regression in melanoma beyond the atypical character of the melanoma cells: distribution of the tumor cells and lymphocytes and the lack of fibrosis in benign lesions (21).

Mechanisms of regression in melanoma are multifactorial and still controversial. It is an inflammatory immune-mediated pathway involving CD8-positive cytotoxic T lymphocytes and NK cells (18). Probably the trigger of regression is a melanocytic antigen (22), destruction of tumor cells being mediated by an inflammatory response of the host (18).

We studied CEACAM1 expression in regressing versus non-regressing thin melanomas, knowing that the phenomenon of regression represents a valuable model for understanding tumor immunity (23).

\section{Materials and methods}

The retrospective study included 53 consecutive cases of thin melanoma, 21 with regression and 32 without. We used the cut-off value for thin melanoma of $2 \mathrm{~mm}$, corresponding to stage 1 and 2 tumors. All the cases were diagnosed in Colentina University Hospital, Department of Pathology in last two years, using histopathological and immunohistochemical techniques. Clinical and histological data were collected and all cases were reexamined by two independent senior pathologists, using Nikon Eclipse E200 microscope (Nikon Instruments Europe B.V., Amsterdam, The Netherlands). Multiple features were analyzed: tumor subtype, thickness, presence or absence of ulceration, mitotic index, Breslow and Clark levels, CEACAM expression.

This study was performed on patients who previously signed a written informed consent and it was approved by the Ethics Committee of Colentina University Hospital. Slides used for research purposes were sectioned after the final diagnosis was signed and did not exhaust the biological material in paraffin blocks, allowing further tests if needed.

All specimens were collected from skin surgical resections of tumors. After surgical resection, samples were immediately immersed in $10 \%$ buffered formalin, then automatically processed using a Leica ASP300 S Fully Enclosed Tissue Processor (Leica Biosystems, Newcastle, UK) and embedded in paraffin. Multiple 3- $\mu \mathrm{m}$ sections were obtained for usual and special stains. After examining hematoxylin and eosin slides, all cases were subjected to appropriate immunohistochemical stains for diagnostic confirmation. In all cases, the final diagnosis was malignant melanoma with Breslow index $<2 \mathrm{~mm}$.

For this study, serial sections from the formalin-fixed, paraffin-embedded blocks were obtained and used for detection of CEACAM1 using three different isoforms of the marker (AA 1-428, extracellular domain, rabbit, cat. no. ABIN1997563; AA 1-428, mouse, clone 8B6E2F4 cat. no. ABIN 1997555; and AA 1-468, full length, mouse, clone 2F6, cat. no. ABIN513717; all from antibodies-online $\mathrm{GmbH}$, Aachen, Germany). Tissue thin sections (3 $\mu \mathrm{m})$ were deparaffinized in xylene, rehydrated with increasing dilutions of ethanol and then with water, and pretreated in a steamer for $30 \mathrm{~min}$ in citrate buffer $(10 \mathrm{mM}, \mathrm{pH}$ 6.0). Slides were washed in Tris-buffered saline (TBS, pH 7.4) and endogenous peroxidase activity was blocked by treatment with peroxidase block, then, incubated with CEACAM1 isoforms (Table I).

Then, slides were examined and membrane positivity for CEACAM-1 was assessed in tumor cells. There were comparatively analyzed tumor cells from areas of partial regression and areas without regression in regressed melanomas and the most intensely positive areas in non-regressed melanoma. Also, we assessed the intensity of the expression in different areas of the tumors (junctional, dermal, invasion front), and classified it on a semi-quantitative scale: 0 , absent; 1 , weak positivity; 2 , moderate positivity; and 3 , intense positivity.

The data were introduced into a data base and statistically analyzed using Microsoft Excel and SPSS (IBM Corp., Released 2015, IBM SPSS Statistics for Windows, version 23.0, Armonk, NY, USA). Welch's t-test and Pearson correlation test were used. P-value $<0.05$ was considered to indicate a statistically significant difference.

\section{Results}

Of our samples, 52 cases were superficial spreading melanomas and just one case (with regression) was an acral-lentiginous 
Table I. Markers used in this study.

\begin{tabular}{lllll}
\hline Epitope & Host & Clonality & Specificity & Supplier \\
\hline AA 1-428 & Rabbit & Polyclonal & Human CEACAM1/CD66 & antibodies-online GmbH \\
AA 1-428 & Mouse & Monoclonal & Human CEACAM1/CD66 & antibodies-online GmbH \\
AA 1-468 & Mouse & Monoclonal & Human CEACAM1/CD66 & antibodies-online GmbH \\
\hline
\end{tabular}

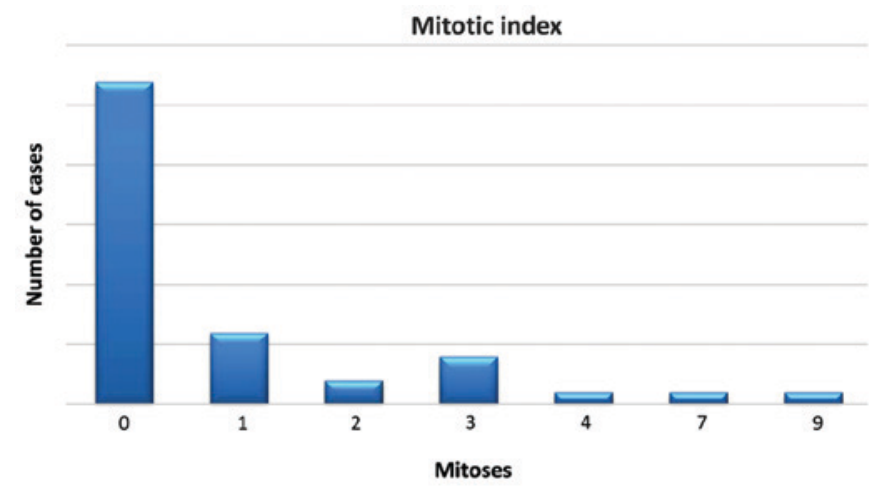

Figure 1. Most thin melanomas have a low mitotic rate.

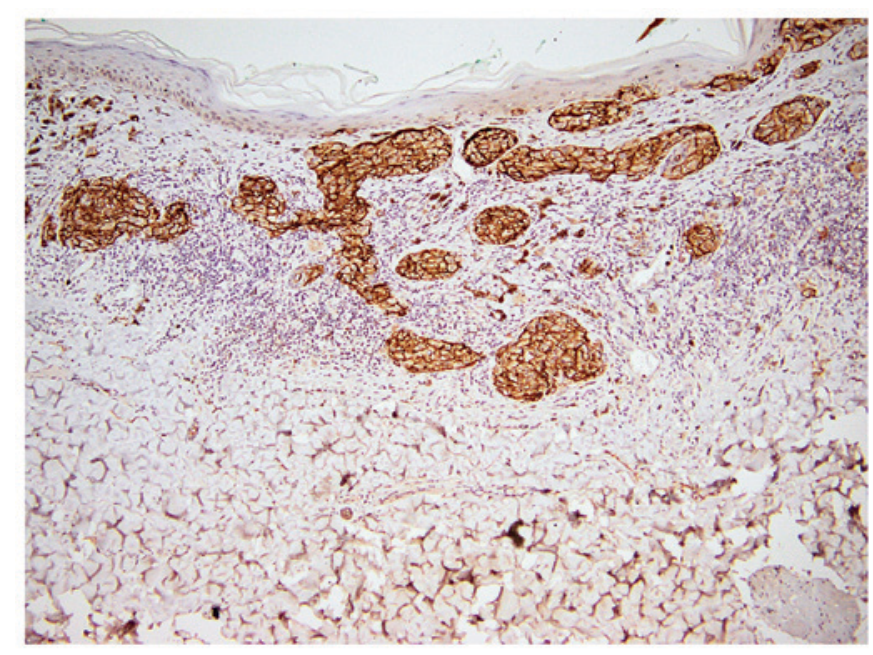

Figure 2. Intense membrane positivity for CEACAM-1 in a melanoma case. CEACAM1. AA 1-428, rabbit; magnification x100.

melanoma. Also, just one case (regressed) had ulceration, all the others lacked this feature.

Median Breslow index of tumors included in the study was $0.688 \mathrm{~mm}$ (with values between 0.16 and $1.75 \mathrm{~mm}$ ), higher in non-regressed melanomas $(0.770 \mathrm{~mm})$ than in regressed ones $(0.660 \mathrm{~mm})$.

Mitotic index was evaluated by quantifying the number of mitotic figures on one square millimeter, in hot spots, the values ranged between 0 and 9 mitotic figures, with an average value of 1 for the entire group (Fig. 1).

All three isoforms of CEACAM-1 had similar reactivity. CEACAM-1 was positive in epithelial cells of sweat ducts and sebaceous glands (used as positive internal control) (Fig. 2).

From the 32 cases of melanoma without regression, only in 3 cases CEACAM-1 was negative in tumor cells, in all the other

\section{Reactivity for CEACAM-1 in melanomas without regression}

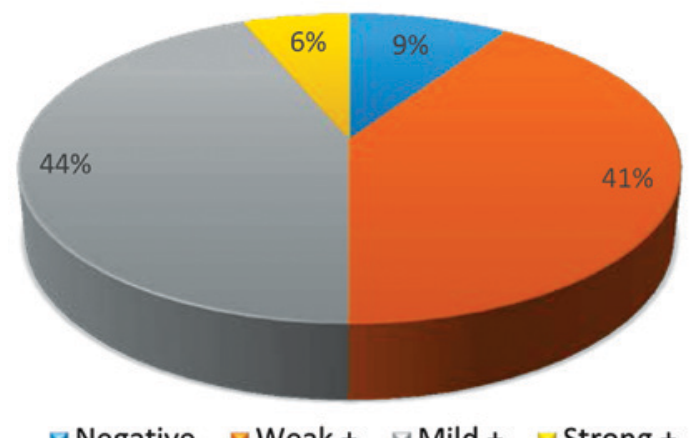

Figure 3. Melanomas without regression are mostly positive for CEACAM-1, with variable intensity of the stain.

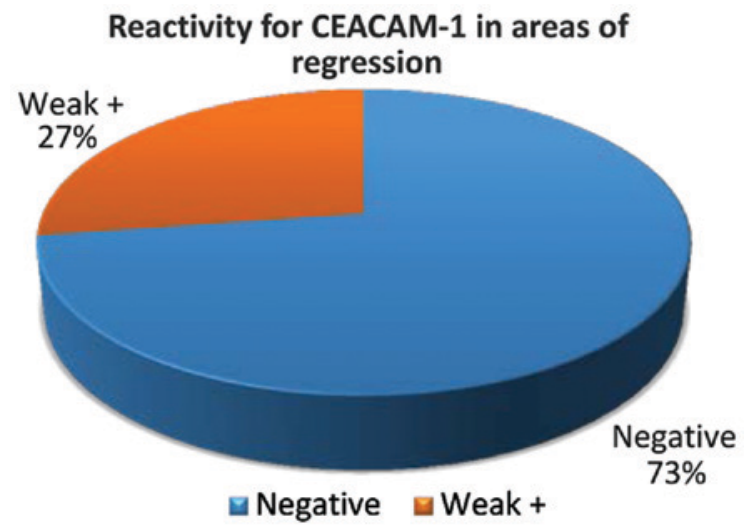

Figure 4. Tumor cells in regressed areas are mostly negative for CEACAM-1.

cases membrane positivity was identified: weak (13 cases), mild (14 cases) and strong (2 cases) (Fig. 3).

In the group of regressed melanomas, a significant difference was observed between remaining (non-regressed) tumor cells from regressed areas (Fig. 4) and tumor cells from non-regressed areas (Fig. 5). While in regressed areas, tumor cells were negative for CEACAM-1 in 16 cases and weakly positive in only 5 cases, in the same group, in non-regressed areas, tumor cells were positive in 19 cases and negative in only 2 cases (Fig. 5).

Statistical correlations showed that the difference of CEACAM-1 reactivity in tumor cells from regressed and non-regressed area (Fig. 6) is highly significant (t-test, $\mathrm{P}<0.0001$ ) (Fig. 7).

Correlation between Breslow index and CEACAM-1 reactivity was significant only in regressed melanomas (Pearson 


\section{Reactivity for CEACAM-1 in non-regressed} areas

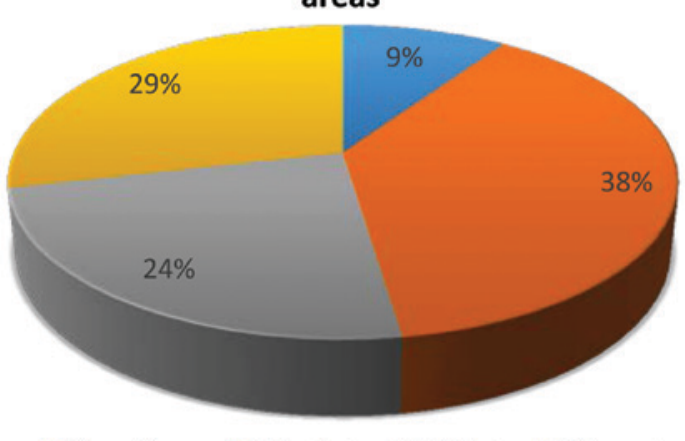

$\square$ Negative $\square$ Weak $+\square$ Mild $+\square$ Strog +

Figure 5. In non-regressed areas of melanomas with regression, tumor cells are mostly positive for CEACAM-1.

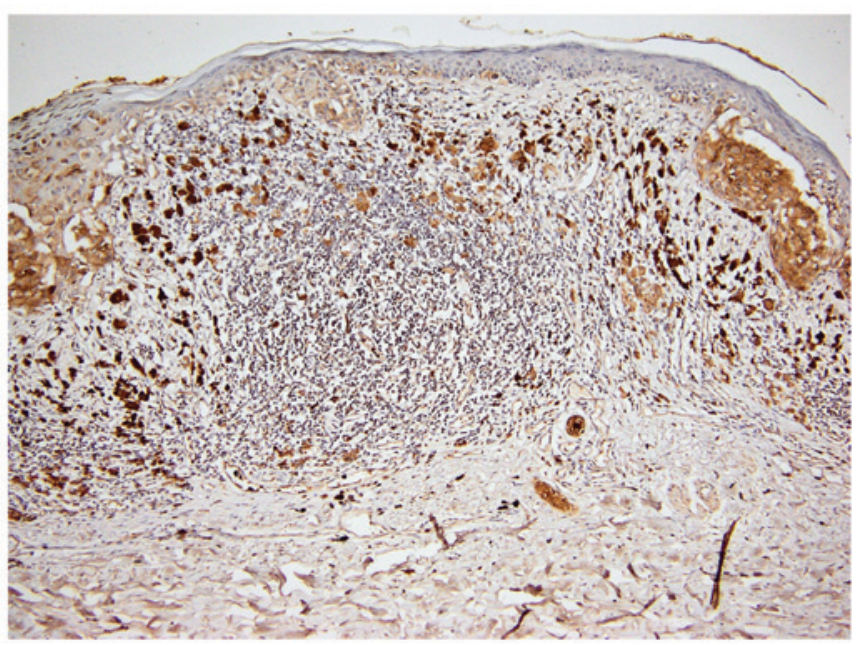

Figure 6. Melanoma with regression. Note mild positivity (arrow) for CEACAM-1 in junctional component and loss of positivity (circle) in the few tumor cells from the regression area. CEACAM1. AA 1-428, mouse; magnification $\mathrm{x} 100$.

\section{Reactivity for CEACAM-1 in melanomas with regression}

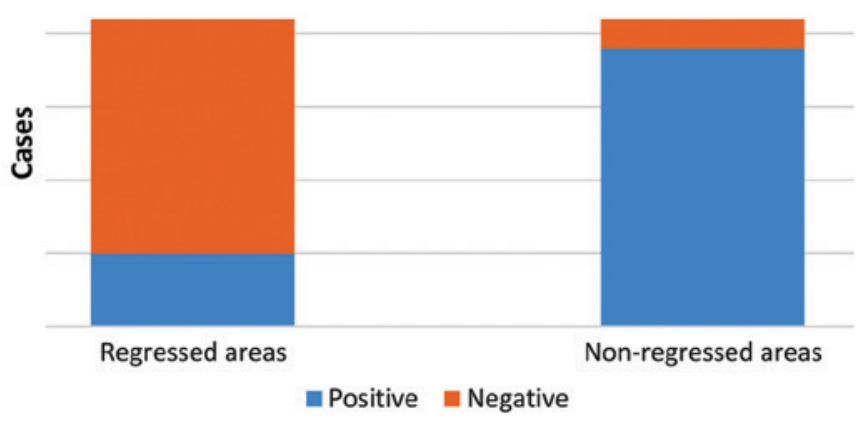

Figure 7. Differences between CEACAM-1 positivity in tumor cells from regressed and non-regressed areas of melanomas with regression.

correlation test, $\mathrm{P}=0.22589$ ). Practically, thicker tumors had stronger global positivity for CEACAM-1 in all studied tumors, but the correlation was not statistically significant in non-regressed melanomas.

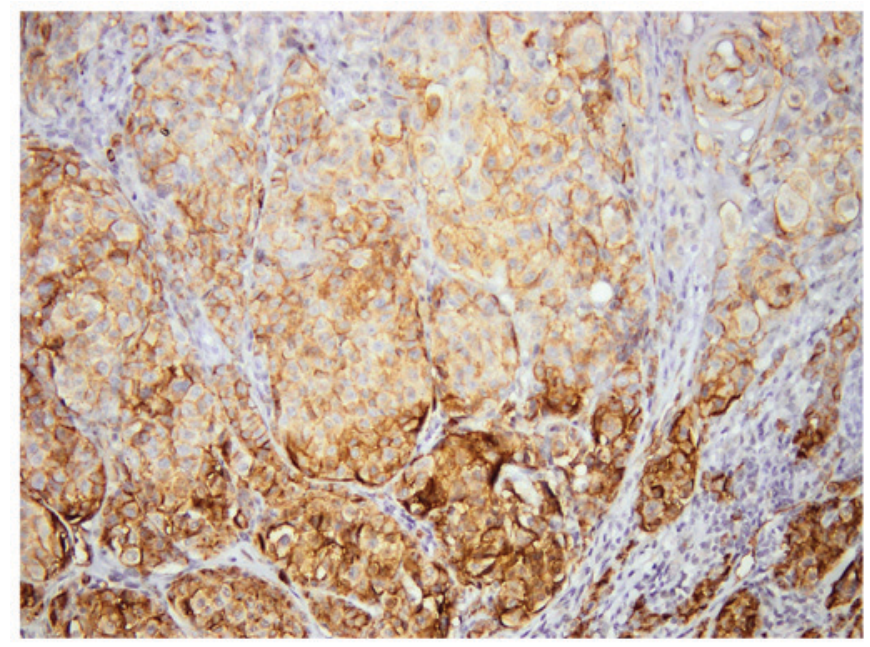

Figure 8. Significant enhancement of CEACAM-1 positivity in the invasion front of a melanoma without regression. Note that the intensity of the staining is higher in the lower half of the image (profound area). CEACAM1. AA 1-428, mouse; magnification x100.

\section{Overexpression of CEACAM-1 in tumor invasion front}

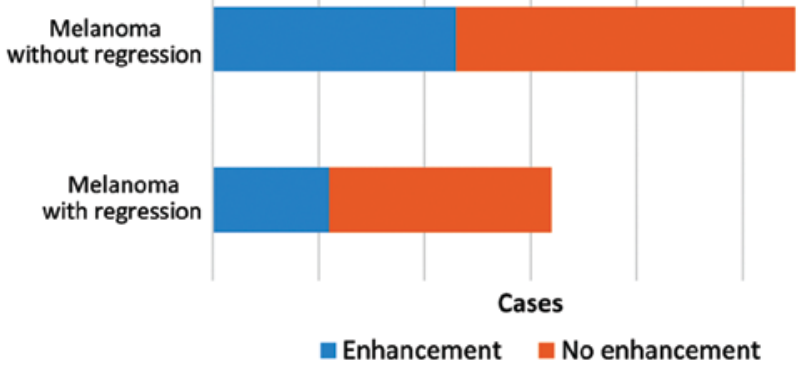

Figure 9. Similar pattern of enhancement of CEACAM-1 expression in melanomas with and without regression.

In 34 cases, representing approximately $64 \%$, we observed a stronger expression of CEACAM-1 in tumor cells from the deep invasion front (Fig. 8). This feature was observed both in regressed melanomas (11 out of 21) and non-regressed tumors (23 out of 32) (Fig. 9).

\section{Discussion}

Superficial spreading melanoma is the most frequent subtype and a less aggressive form of the tumor. Since it has a long period of radial growth, without invasion, it is the most probable tumor to be diagnosed as thin melanoma (24). In our study, the vast majority of thin melanomas were superficial spreading type, with nodular growth and invasion. Ulceration is also a rare feature in thin melanomas but is considered a poor prognosis factor related with positive sentinel node and significant risk of metastasis (25-27). In our group only one tumor was ulcerated, a regressed superficial spreading melanoma with high mitotic index (9) and Breslow level $1.4 \mathrm{~mm}$, confirming the aggressive potential of this proliferation. In this particular tumor, CEACAM-1 was weakly positive in regressed areas and strongly positive in non-regressed areas. 
Considering that CEACAM-1 is a membrane protein that promotes invasion and metastasis in melanoma, this characteristic confirms the fact that this tumor could have a poor outcome (28). Immunohistochemical testing for CEACAM-1 in melanomas could help stratifying patients according to their risk to progression and represents an interesting feature that can represent not only a therapeutical target, but also a morphologic trait that helps personalizing the treatment and surveillance in melanoma patients.

CEACAM-1 is positive in the vast majority of cases of melanoma, but our study identified a significant difference between the intensity of reaction in various areas of the tumor. Melanomas without regression and areas without regression from regressed melanomas showed a similar profile of CEACAM-1 positivity, while melanomas with regression exhibited a very low rate of CEACAM-1 positivity in remaining tumor cells form regressed areas. This specific immunophenotype implies that these tumor cells have special characteristics and lack a feature that confers their counterparts from non-regressed areas with potential of invasion. This observation raises two hypotheses: either regression affects only some tumor cells, less aggressive, with special features, or inflammatory cells and inflammatory mediators are inducing changes in tumor cell phenotype and behaviour.

Some studies demonstrated that CEACAM-1 enhances vascular neo-proliferation and reduces cell sensitivity to hypoxia, mechanism involved in inflammatory-driven tumor regression (29,30). These data suggest that CEACAM-1 defective cells are more sensitive to hypoxia and less capable of stimulating tumor angiogenesis. Also, CEACAM-1 expression modulates apoptosis (10). These characteristics make tumor cells more sensitive to microenvironment changes induced by inflammation and more prone to be destroyed by T lymph cells and NK cells. Melanoma is a poor immunogenic tumor in advanced stages leading to immune suppression and consequently to immune tolerance by so-called 'immunosculpting' process $(31,32)$.

On the other hand, our study confirms that CEACAM-1 is overexpressed in cells from the invasion front, in regressed and non-regressed melanomas. This is related in all studies with a decrease of cell-adhesion and a higher capacity of stromal invasion $(28,33)$. Overexpression of CEACAM-1 in front invasion cells represents an important dysregulation of cell adhesion, enhancing the transition from radial growth phase (with good prognosis and reduced risk of metastasis) to vertical growth phase (with high risk of metastasis and poor prognosis) $(33,34)$. CEACAM-1 may intervene in mechanisms involved in immune surveillance escaping $(35,36)$ and it is a possible candidate to be a monitoring biomarker and a possible target; supplementary investigations are necessary $(3,37,38)$.

CEACAM1 is a valuable marker in melanoma that can be used for a more complete description of tumor features related to invasiveness and aggressive behavior. It is more intensely positive in thick melanomas and in invasion front, indicating that CEACAM1-positive cells have a higher potential of invasion and metastasis.

Also, there is significant loss of CEACAM1 expression in melanoma cells from areas of regression indicating that regression is not only the result of inflammation, but also of some specific characteristics of some tumor cells that make them more sensitive to the cytotoxic lymphocyte action.
Monoclonal antibodies against CEACAM1 can induce loss of CEACAM1 expression in melanoma cells and enhance antitumor effect of patients' immune system and has been evaluated in a phase 1 study for safety and tolerability (NCT02346955) completed in 2017 (https://clinicaltrials.gov/ ct2/show/NCT02346955; identification no. NCT02346955). Phase 2 clinical trials will further evaluate the adequate doses. CEACAM1 is a promising therapeutic target, since loss of expression in tumor cells seems to stimulate regression and inhibit vertical growth and invasion.

\section{Acknowledgments}

The authors would like to thank physicians from Colentina Hospital and Dermato-oncology Excellence Centre (Bucharest, Romania) for diagnosing, treating and monitoring the patients with cutaneous melanoma included in this research.

\section{Funding}

This study was partially supported by grants of Romanian Ministry of Research and Innovation, CCCDI-UEFISCDI, project nos. 61PCCDI/2018 PN-III-P1-1.2-PCCDI-2017-0341 and CNCS-UEFISCDI and 183/2017PN-III-P4ID-PCE-2016-0641, within PNCDI-III.

\section{Availability of data and materials}

The datasets used and/or analyzed during the current study are available from the corresponding author on reasonable request.

\section{Authors' contributions}

LN and SZ contributed to the concept and draft of this study. LN, SZ, CP, MC and DI designed the study and implemented each step of the research. LN, SZ, CP, MC, ABa, LJ, LS and PS examined the archives and identified the cases included in the study, examined the slides and collected pathological information. RN, ABr and GT clinically diagnosed the patients, obtained the informed consent, harvested tissue samples. All authors participated in statistical analysis, finding and interpreting the results, drafting the manuscript and revising it critically for important intellectual content. All authors read and approved the final manuscript.

\section{Ethics approval and consent to participate}

This research follows international and national regulations in accordance with the Declaration of Helsinki. It was approved by the Ethics Committee of Colentina University Hospital (Bucharest, Romania). All patients signed an informed consent before being included in this study. Their personal data were maintained confidential during this research, only their medical doctors (dermatologists and pathologists) having access to their identity.

\section{Patient consent for publication}

Not applicable. 


\section{Competing interests}

All authors declare that they have no competing interests.

\section{References}

1. Fiori V, Magnani $M$ and Cianfriglia $M$ : The expression and modulation of CEACAM1 and tumor cell transformation. Ann Ist Super Sanita 48: 161-171, 2012.

2. Beauchemin N, Draber P, Dveksler G, Gold P, Gray-Owen S, Grunert F, Hammarström S, Holmes KV, Karlsson A, Kuroki M, et al: Redefined nomenclature for members of the carcinoembryonic antigen family. Exp Cell Res 252: 243-249, 1999.

3. Dankner M, Gray-Owen SD, Huang YH, Blumberg RS and Beauchemin N: CEACAM1 as a multi-purpose target for cancer immunotherapy. OncoImmunology 6: e1328336, 2017.

4. Neagu M: The immune system - a hidden treasure for biomarker discovery in cutaneous melanoma. Adv Clin Chem 58: 89-140, 2012

5. Sapoznik S, Ortenberg R, Schachter J and Markel G: CEACAM1 in malignant melanoma: A diagnostic and therapeutic target. Curr Top Med Chem 12: 3-10, 2012

6. Sivan S, Suzan F, Rona O, Tamar H, Vivian B, Tamar P, Jacob S, Gal M and Michal L: Serum CEACAM1 correlates with disease progression and survival in malignant melanoma patients. Clin Dev Immunol 2012: 290536, 2012 .

7. Thies A, Berlin A, Brunner G, Schulze HJ, Moll I, Pfüller U, Wagener C, Schachner M, Altevogt P and Schumacher U: Glycoconjugate profiling of primary melanoma and its sentinel node and distant metastases: Implications for diagnosis and pathophysiology of metastases. Cancer Lett 248: 68-80, 2007.

8. Khatib N, Pe'er J, Ortenberg R, Schachter J, Frenkel S, Markel G and Amer R: Carcinoembryonic antigen cell adhesion molecule-1 (CEACAM1) in posterior uveal melanoma: Correlation with clinical and histological survival markers. Invest Ophthalmol Vis Sci 52: 9368-9372, 2011

9. Nittka S, Günther J, Ebisch C, Erbersdobler A and Neumaier M: The human tumor suppressor CEACAM1 modulates apoptosis and is implicated in early colorectal tumorigenesis. Oncogene 23 9306-9313, 2004.

10. Turcu G, Nedelcu RI, Ion DA, Brînzea A, Cioplea MD, Jilaveanu LB and Zurac SA: CEACAM1: Expression and role in melanocyte transformation. Dis Markers 2016: 9406319, 2016.

11. Markel G, Seidman R, Stern N, Cohen-Sinai T, Izhaki O, Katz G, Besser M, Treves AJ, Blumberg RS, Loewenthal R, et al: Inhibition of human tumor-infiltrating lymphocyte effector functions by the homophilic carcinoembryonic cell adhesion molecule 1 interactions. J Immunol 177: 6062-6071, 2006.

12. Ortenberg R, Sapoznik S, Zippel D, Shapira-Frommer R, Itzhaki O, Kubi A, Zikich D, Besser MJ, Schachter J and Markel G: Serum CEACAM1 elevation correlates with melanoma progression and failure to respond to adoptive cell transfer immunotherapy. J Immunol Res 2015: 902137, 2015.

13. Kluger HM, Hoyt K, Bacchiocchi A, Mayer T, Kirsch J, Kluger Y, Sznol M, Ariyan S, Molinaro A and Halaban R: Plasma markers for identifying patients with metastatic melanoma. Clin Cancer Res 17: 2417-2425, 2011.

14. Ortenberg R, Sapir Y, Raz L, Hershkovitz L, Ben Arav A, Sapoznik S, Barshack I, Avivi C, Berkun Y, Besser MJ, et al: Novel immunotherapy for malignant melanoma with a monoclonal antibody that blocks CEACAM1 homophilic interactions. Mol Cancer Ther 11: 1300-1310, 2012.

15. Ebrahimnejad A, Streichert T, Nollau P, Horst AK, Wagener C, Bamberger AM and Brümmer J: CEACAM1 enhances invasion and migration of melanocytic and melanoma cells. Am J Pathol 165: 1781-1787, 2004.

16. Gambichler T, Grothe S, Rotterdam S, Altmeyer P and Kreuter A: Protein expression of carcinoembryonic antigen cell adhesion molecules in benign and malignant melanocytic skin lesions. Am J Clin Pathol 131: 782-787, 2009.

17. Gimotty PA and Guerry D: Prognostication in thin cutaneous melanomas. Arch Pathol Lab Med 134: 1758-1763, 2010.

18. Aung PP, Nagarajan P and Prieto VG: Regression in primary cutaneous melanoma: Etiopathogenesis and clinical significance. Lab Invest 97: 657-668, 2017

19. Zurac S, Neagu M, Constantin C, Cioplea M, Nedelcu R, Bastian A, Popp C, Nichita L, Andrei R, Tebeica T, et al: Variations in the expression of TIMP1, TIMP2 and TIMP3 in cutaneous melanoma with regression and their possible function as prognostic predictors. Oncol Lett 11: 3354-3360, 2016.
20. Zurac S, Negroiu G, Petrescu S, Andrei R, Tebeica T, Popp C, Musţată R, Neagu M, Constantin C, Solovan C, et al: Spectrum of morphologic alterations of regression in cutaneous melanoma - potential for improving disease prognosis. Rom J Intern Med 50: 145-153, 2012.

21. Nedelcu RI, Zurac SA, Brînzea A, Cioplea MD, Turcu G, Popescu R, Popescu CM and Ion DA: Morphological features of melanocytic tumors with depigmented halo: Review of the literature and personal results. Rom J Morphol Embryol 56 (Suppl 2): 659-663, 2015.

22. Saleh FH, Crotty KA, Hersey P and Menzies SW: Primary melanoma tumour regression associated with an immune response to the tumour-associated antigen melan-A/MART-1. Int J Cancer 94: 551-557, 2001

23. Lupu M, Caruntu A, Caruntu C, Papagheorghe LM, Ilie MA, Voiculescu V, Boda D, Constantin C, Tanase C, Sifaki M, et al: Neuroendocrine factors: The missing link in non-melanoma skin cancer (Review). Oncol Rep 38: 1327-1340, 2017.

24. Shashanka R and Smitha BR: Head and neck melanoma. ISRN Surg 2012: 948302, 2012.

25. Rubinstein JC, Han G, Jackson L, Bulloch K, Ariyan S, Narayan D, Rothberg BG and Han D: Regression in thin melanoma is associated with nodal recurrence after a negative sentinel node biopsy. Cancer Med 5: 2832-2840, 2016.

26. Diaconeasa A, Boda D, Solovan C, Enescu DM, Vîlcea AM and Zurac S: Histopathologic features of Spitzoid lesions in different age groups. Rom J Morphol Embryol 54: 51-62, 2013.

27. Blendea A, Georgescu CV, Ţolea I, Brănişteanu DE and Costache A: An unusual cutaneous malignant melanoma arised de novo: A case report. Rom J Morphol Embryol 56: 1217-1221, 2015.

28. Ortenberg R, Galore-Haskel G, Greenberg I, Zamlin B, Sapoznik S, Greenberg E, Barshack I, Avivi C, Feiler Y, Zan-Bar I, et al: CEACAM1 promotes melanoma cell growth through Sox-2. Neoplasia 16: 451-460, 2014.

29. Ludewig P, Flachsbarth K, Wegscheid C, Tiegs G, Richard G, Wagener C, Bartsch U and Horst AK: CEACAM1 confers resistance toward oxygen-induced vessel damage in a mouse model of retinopathy of prematurity. Invest Ophthalmol Vis Sci 55: 7950-7960, 2014.

30. Horst AK, Ito WD, Dabelstein J, Schumacher U, Sander H, Turbide C, Brümmer J, Meinertz T, Beauchemin N and Wagener C: Carcinoembryonic antigen-related cell adhesion molecule 1 modulates vascular remodeling in vitro and in vivo. J Clin Invest 116: 1596-1605, 2006.

31. Bulman A, Neagu M and Constantin C: Immunomics in skin cancer - improvement in diagnosis, prognosis and therapy monitoring. Curr Proteomics 10: 202-217, 2013.

32. Boda D: Cellomics as integrative omics for cancer. Curr Proteomics 10: 237-245, 2013.

33. Thies A, Moll I, Berger J, Wagener C, Brümmer J, Schulze HJ, Brunner $G$ and Schumacher U: CEACAM1 expression in cutaneous malignant melanoma predicts the development of metastatic disease. J Clin Oncol 20: 2530-2536, 2002.

34. Caruntu C, Boda D, Constantin C, Caruntu A and Neagu M: Catecholamines increase in vitro proliferation of murine $\mathrm{B} 16 \mathrm{~F} 10$ melanoma cells. Acta Endocrinol (Copenh) 10: 545-558, 2014.

35. Markel G, Seidman R, Cohen Y, Besser MJ, Sinai TC, Treves AJ, Orenstein A, Berger R and Schachter J: Dynamic expression of protective CEACAM1 on melanoma cells during specific immune attack. Immunology 126: 186-200, 2009.

36. Markel G, Ortenberg R, Seidman R, Sapoznik S, Koren-Morag N, Besser MJ, Bar J, Shapira R, Kubi A, Nardini G, et al: Systemic dysregulation of CEACAM1 in melanoma patients. Cancer Immunol Immunother 59: 215-230, 2010.

37. Neagu M, Constantin C and Tanase C: Immune-related biomarkers for diagnosis/prognosis and therapy monitoring of cutaneous melanoma. Expert Rev Mol Diagn 10: 897-919, 2010.

38. Neagu M, Caruntu C, Constantin C, Boda D, Zurac S, Spandidos DA and Tsatsakis AM: Chemically induced skin carcinogenesis: Updates in experimental models (Review). Oncol Rep 35: 2516-2528, 2016.

This work is licensed under a Creative Commons Attribution-NonCommercial-NoDerivatives 4.0 International (CC BY-NC-ND 4.0) License. 